\title{
Importance de la cavitation lors de la fissuration en fatigue du caoutchouc naturel chargé
}

\author{
Jean-Benoît Le Cam ${ }^{1,2}$, Franck Pérocheau ${ }^{2}$, Bertrand Huneau ${ }^{1}$, Erwan Verron ${ }^{1, a}$ \\ ET LAURENT GORNET ${ }^{1}$ \\ 1 Institut de Recherche en Génie Civil et Mécanique, École Centrale de Nantes, BP 92101, 44321 Nantes Cedex 03, France \\ 2 Allevard Rejna Autosuspensions, 201 rue Sin Le Noble, 59506 Douai Cedex, France
}

Reçu le 6 novembre 2003, accepté le 21 novembre 2005

\begin{abstract}
Résumé - Ce travail présente des analyses fractographiques de fissures de fatigue dans le caoutchouc naturel chargé aux noirs de carbone. Afin d'identifier les mécanismes de propagation de fissure, il est nécessaire de connaître l'état de la microstructure autour du fond de fissure. Nous avons donc mis au point une technique permettant de micro-découper le fond de fissure lors d'observations au microscope électronique à balayage (MEB). Cette technique utilise simultanément l'énergie mécanique apportée par l'étirement statique de l'éprouvette dans le MEB et l'énergie fournie par la concentration du faisceau électronique du MEB sur la zone d'observation. Les résultats de ces observations montrent que l'endommagement par fatigue au fond de fissure est dû à la cavitation engendrée par la décohésion entre la matrice polymère et les oxydes.
\end{abstract}

Mots clés : Caoutchouc / fatigue / endommagement / propagation de fissure / cavitation / fractographie / micro-découpe

\begin{abstract}
Cavitation influence on fatigue crack growth in filled natural rubber. The aim of this present paper is the fractographic analysis of cracks in a carbon black filled natural rubber under fatigue loading conditions. In order to determine the crack growth mechanism, the microstructural state behind the crack tip should be analysed. Here, an experimental method that consists in cutting the crack tip during its observation with a scanning electron microscope is proposed. The crack tip opening is performed using two different energetic sources in order to break cross-links between the macromolecules. The first source is mechanical: the sample is stretched on a simple tensile apparatus. The second one is the electronic beam. Observation results indicate that cavitation is the fatigue damage which drives crack growth. The cavitation mechanism is initiated by the decohesion of the oxides and rubber matrix.
\end{abstract}

Key words: Rubber / fatigue / damage / crack growth / cavitation / fractography / micro-cut

\section{Introduction}

La plupart des études menées sur la propagation de fissure dans les élastomères emploient des approches purement mécaniques, souvent issues de l'étude des métaux; celles-ci utilisent le plus souvent le concept d'énergie de déchirement [1]. Cette approche phénoménologique fournit des résultats intéressants, mais ne permet pas de prédire de façon systématique les chemins de propagation de fissure dans les pièces élastomères. C'est pourquoi, la détermination des mécanismes physiques liés à la fatigue mécanique apparaît nécessaire afin d'enrichir ce type d'approche.

\footnotetext{
a Auteur correspondant : erwan.verron@ec-nantes.fr
}

Pour comprendre les mécanismes microscopiques mis en jeu lors de la propagation de fissures dans les matériaux, des analyses fractographiques de faciès de rupture réalisées par microscopie électronique à balayage (MEB) sont menées pour mettre en évidence les évènements caractéristiques du type de sollicitation et du matériau considéré. Ces observations précisent la source de l'initiation de la fissure et retracent le chemin de propagation. Dans les polymères à l'état vitreux, la propagation de fissure a été plus largement étudiée et des microcinétiques de fissuration ont déjà été proposées [2-5]. En revanche, pour les élastomères, les études menées jusqu'à présent ne permettent pas d'expliquer de manière claire et unanime le mécanisme de fissuration [6-10]. 
Afin de comprendre et de représenter le (ou les) mécanisme(s) de propagation de la fissure en grandes déformations, il nous paraît nécessaire de connaître l'état microstructural de la matière au fond de fissure. Pour cela, nous avons mis au point une technique qui permet d'identifier le dommage prépondérant en fatigue dans le caoutchouc naturel chargé.

\section{Création d'une fissure de fatigue}

La fissure que nous avons étudiée est préalablement créée par un essai de fatigue.

\section{1 Éprouvette}

La géométrie d'éprouvette choisie est celle proposée par Beatty [11]. Comme le montre la figure 1, elle est axisymétrique et admet un rayon de courbure important, ce qui lui vaut le nom d'éprouvette Diabolo. Ce rayon permet d'obtenir une déformation locale au centre supérieure à la déformation nominale imposée à l'éprouvette, ce qui permet de localiser la fissure de fatigue en traction relaxante et de réduire le temps d'essai.

\subsection{Matériau}

Le matériau utilisé est un caoutchouc naturel chargé au noir de carbone. Le tableau 1 résume sa composition chimique ainsi que quelques caractéristiques mécaniques. Le dosage des composants est réalisé en pce ou parties pour cent d'élastomère en masse. Le procédé d'élaboration est le moulage par injection.

\subsection{Conditions de chargement}

Afin de s'assurer de la présence des dommages caractéristiques de la réponse en fatigue du matériau en fond de fissure, l'éprouvette est préalablement soumise à un chargement cyclique relaxant en déplacement imposé (retour à déplacement nul), correspondant à une élongation de 1,33. L'expérience est menée jusqu'à l'obtention d'une fissure de l'ordre de $5 \mathrm{~mm}$ en surface dans l'état non-déformé. La figure 2 montre que la fissure est apparue au centre de l'éprouvette et à proximité du plan de joint. Cette fissure, générée en fatigue, permet d'obtenir une microstructure représentative du dommage de fatigue au voisinage du front de fissure. Pour éviter de superposer un endommagement thermique au endommagement mécanique [12], la vitesse de déformation a été contrôlée pendant l'essai de manière à ne pas élever la température de peau de plus de $20^{\circ} \mathrm{C}$, la température de l'enceinte de l'essai étant stabilisée à $23^{\circ} \mathrm{C}$.

Comme le montre la figure 3, l'éprouvette est ensuite montée sur un dispositif expérimental de traction statique composé de tiges filetées et d'écrous pour écarter les inserts. Les axes des tiges filetées sont positionnés

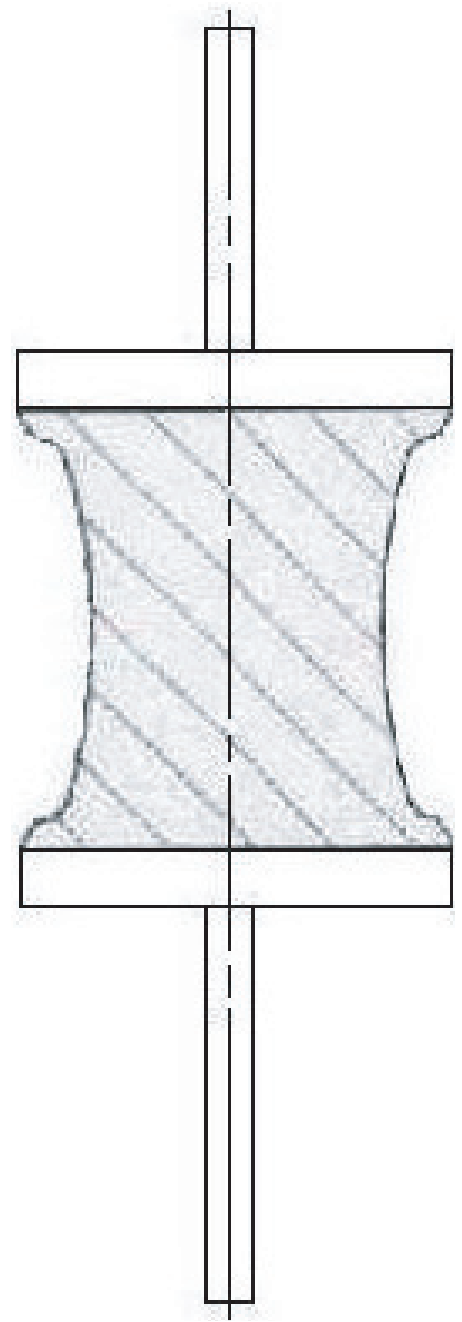

Fig. 1. Éprouvette Diabolo.

Tableau 1. Formulation et caractéristiques mécaniques du matériau.

\begin{tabular}{lr}
\hline Formulation (pce) & \\
\hline NR & 100,00 \\
Oxyde de zinc & 9,85 \\
Plastifiant & 3,00 \\
Noir de carbone & 34,00 \\
Acide stéarique & 3,00 \\
Antioxydant & 2,00 \\
Accélérateurs & 4,00 \\
\hline Caractéristiques mécaniques & \\
\hline Densité & 1,13 \\
Dureté shore A & 58,00 \\
Contrainte à rupture (Mpa) & 22,90 \\
Allongement à rupture (\%) & 635,00 \\
\hline
\end{tabular}

de manière à laisser la fissure accessible au faisceau électronique du MEB. Pour observer le voisinage et le fond la fissure, on impose à l'éprouvette la même élongation qu'en fatigue $(1,33)$. La figure 4 montre l'éprouvette étirée, avec la fissure ouverte de $5 \mathrm{~mm}$. 


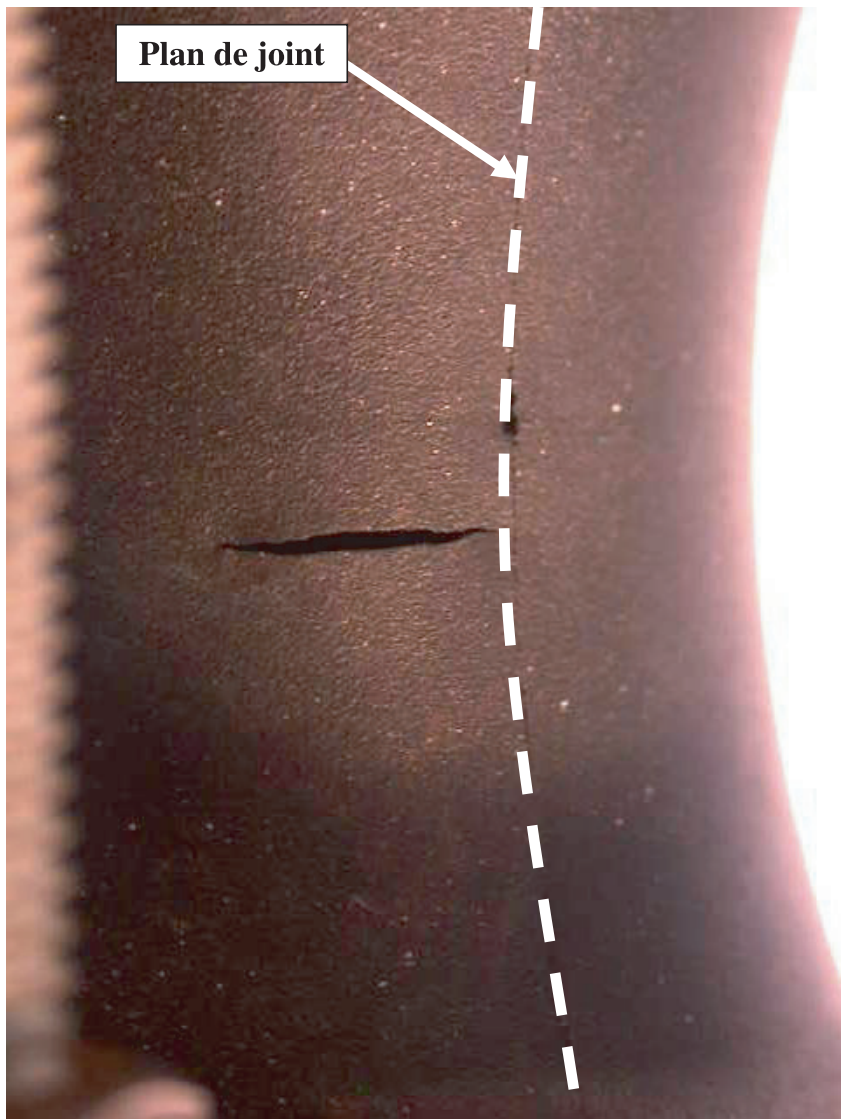

Fig. 2. Éprouvette fissurée.

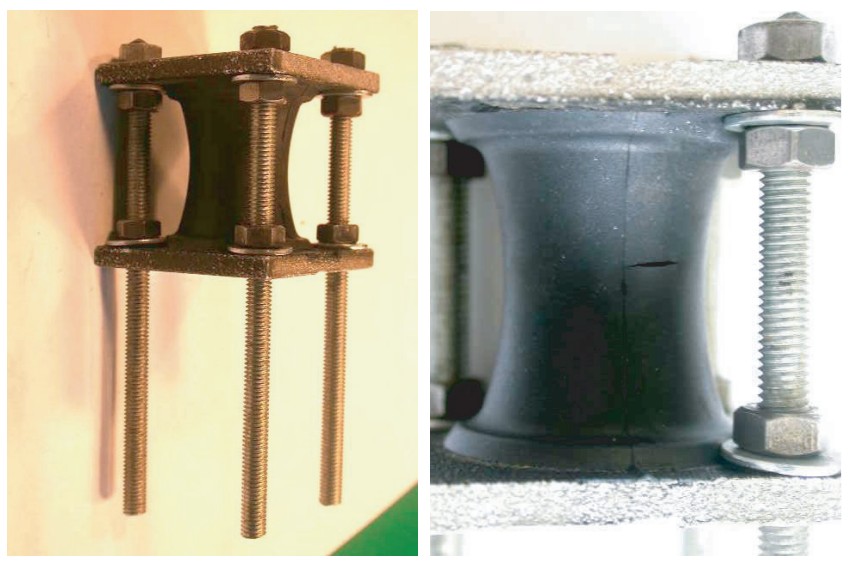

Fig. 3. Montage de mise en tension, la fissure est rendue accessible pour l'observation au MEB.

Des essais de fatigue complémentaires de traction relaxante ont été réalisés mais cette fois-ci jusqu'à rupture, pour des élongations maximales égales à 1,33 et 1,67.

\section{Description de la fissure}

\subsection{Méthode d'observation}

L'éprouvette fissurée et étirée est dépolluée dans un bain de solution neutre soumis aux ultrasons. Le matériau

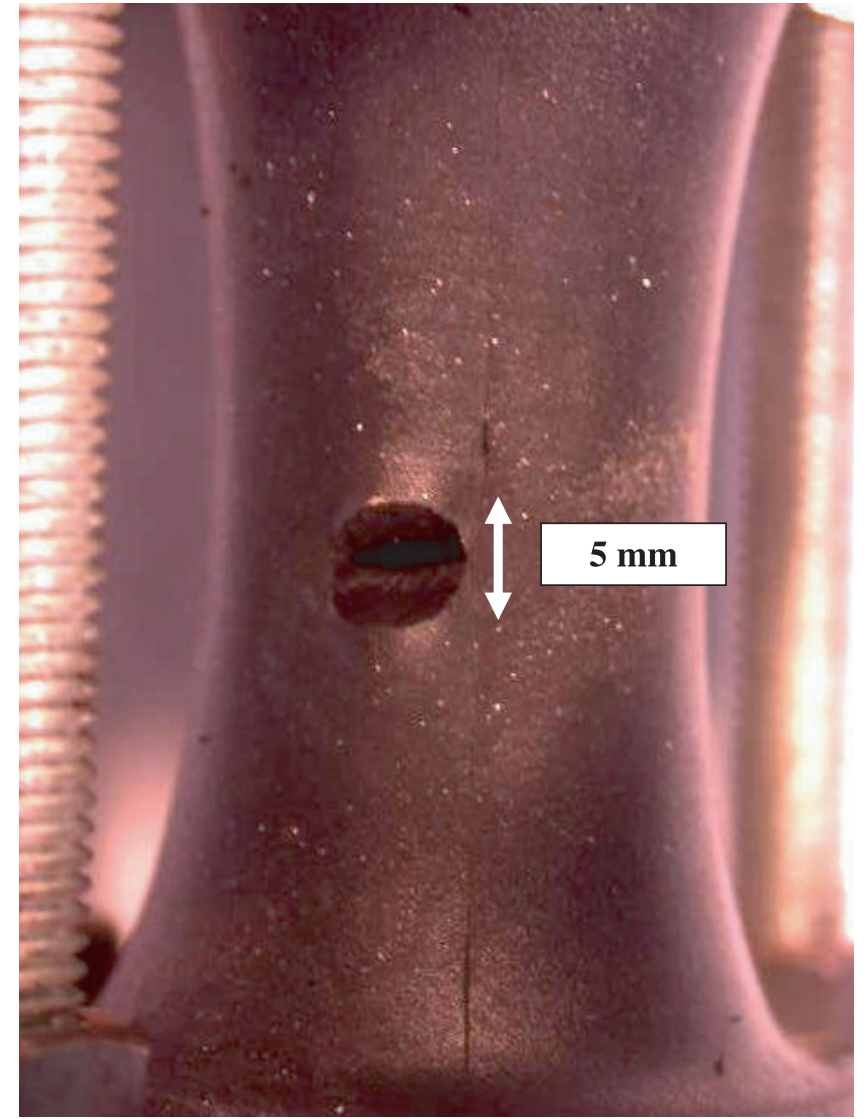

Fig. 4. Ouverture de la fissure (élongation de 1,33).

n'étant pas suffisamment conducteur, l'éprouvette étirée est métallisée à l'or (couche de $90 \mathrm{~nm}$ d'épaisseur). Elle est ensuite introduite dans la chambre du MEB pour y être analysée à partir des électrons secondaires. Un dispositif de micro-analyse X, couplé au MEB, permet de recenser les éléments présents sur les surfaces analysées.

\subsection{Résultats}

Avant de décrire la fissure, il est important de détailler son voisinage, et en particulier les états de surface des zones voisines, représentatifs de leurs étirements respectifs.

\subsubsection{Description générale}

La figure 5 représente la vue schématique du cliché de la figure 4. Quatre zones d'état de surface différent sont présentes autour de la fissure :

- La zone A est la moins étirée; elle bénéficie du rétreint des chaînes rompues lors de la propagation;

- La zone $\mathrm{B}$ est très étirée, et comme le montre la figure 6 , de nombreuses craquelures sont présentes ;

- La zone $\mathrm{C}$ correspond à une surface peu étirée puisque la section de l'éprouvette dans cette zone est croissante jusqu'aux inserts ; 


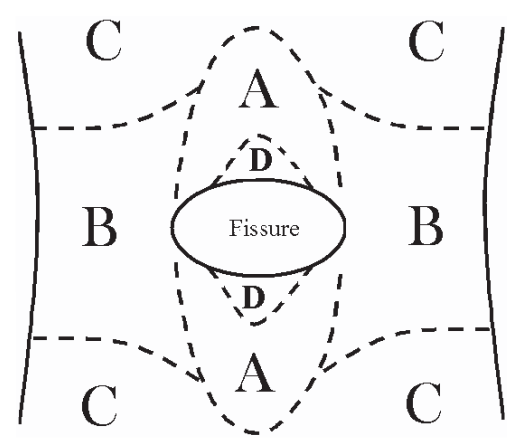

Fig. 5. Trois zones d'état de surface différent autour de la fissure.

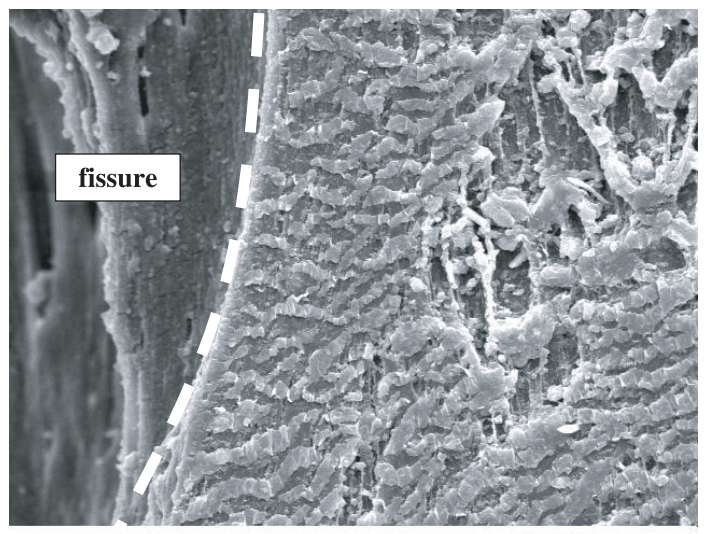

$25 \mu \mathrm{m}$

Fig. 6. Zone B (grossissement $900 \times)$.

- La zone D se situe de part et d'autre de la fissure. Des micro-cavités de surface sont présentes jusqu'à $0,2 \mathrm{~mm}$ du bord de la fissure, comme le montre la figure 7 . Ces micro-cavités sont de l'ordre de quelques centaines de nanomètres. Ces cavités peuvent correspondre à l'empreinte d'ingrédients solides peu liés (physiquement et/ou chimiquement) à la matrice élastomère et éjectés lors de l'essai de fatigue, particulièrement lors de l'apparition de la fissure en surface.

\subsubsection{Détail de la fissure}

Le cliché de la figure 8a montre la fissure dans son intégralité. Les différentes surfaces repérées sont reportées sur le schéma de la vue de profil figure $8 \mathrm{~b}$. Le fond de la fissure est une surface assez lisse, et les bords, correspondant à des surfaces rompues, sont rugueux. La rugosité de ces surfaces est due à la présence d'arrachements de matière. La zone E définit la frontière entre la partie lisse et la partie rugueuse. Un grossissement de cette zone est présenté sur le cliché de la figure 9. Les arrachements de la partie rugueuse sont de plus en plus étirés vers la partie lisse et des filaments apparaissent entre eux. Ces arrachements, présents sur les surfaces rompues, sont caractéristiques des faciès de rupture des caoutchoucs naturels sollicités en fatigue sous conditions de traction relaxante.

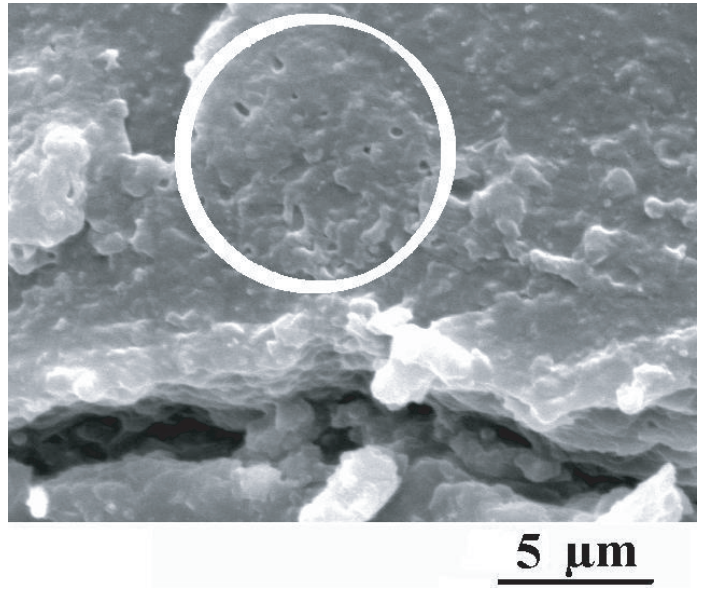

Fig. 7. Micro-cavités de la zone D (bord de fissure en bas).
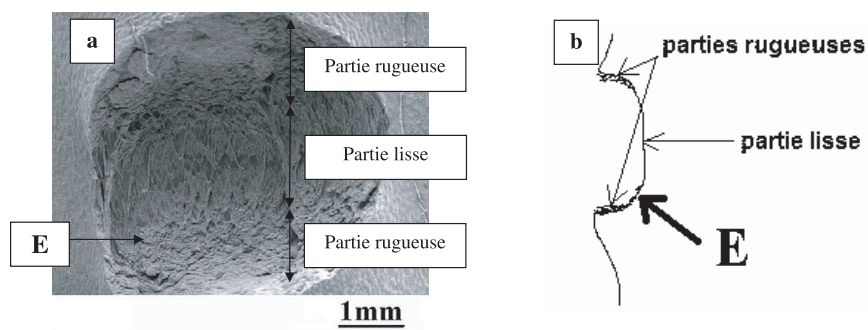

Fig. 8. (a) Fissure de $5 \mathrm{~mm}$ pour une élongation de 1,33; (b) profil de la fissure. Des parties rugueuses jouxtent le fond lisse de la fissure.

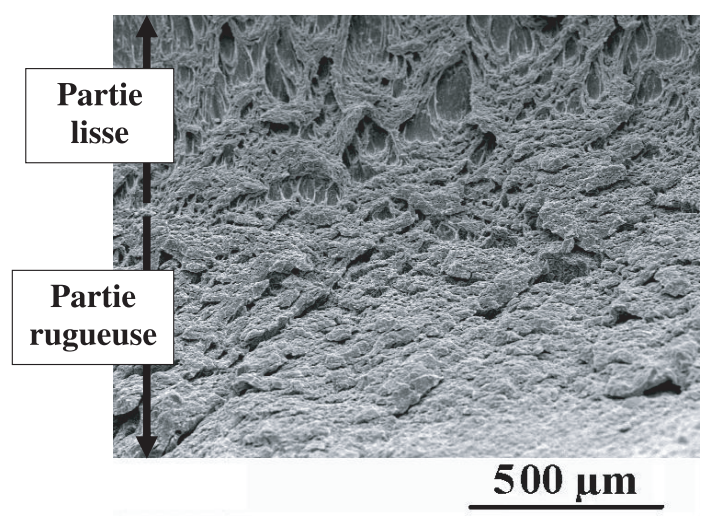

Fig. 9. Zone E de la figure 8 : interface entre la partie rugueuse et la partie lisse.

La figure 10 montre les longs filaments formés par la matière étirée qui constitue le fond de la fissure et la figure 11 est un grossissement de la zone la plus étirée, au centre de la fissure. Cette dernière montre qu'il y a des oxydes entre les filaments (particules blanches). Ces oxydes se trouvent dans des zones ellipsoïdales fortement étirées.

\section{Identification du dommage}

Comme nous venons de le voir précédemment, l'observation du fond de fissure met en évidence les différents 


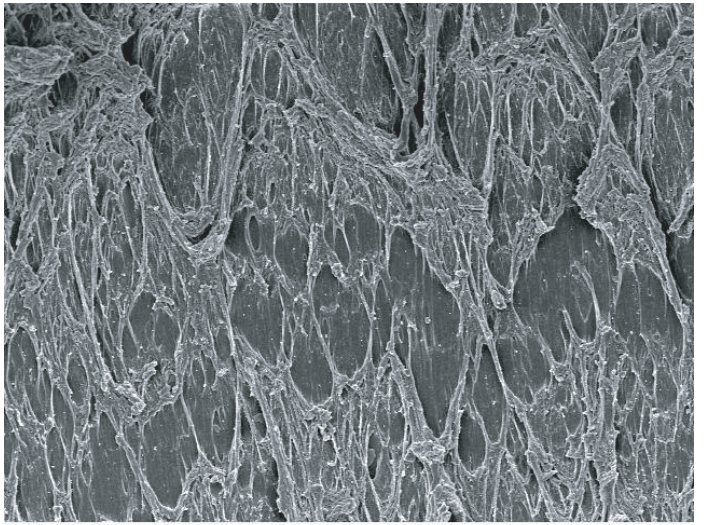

$500 \mu \mathrm{m}$

Fig. 10. Filaments au fond de la fissure.

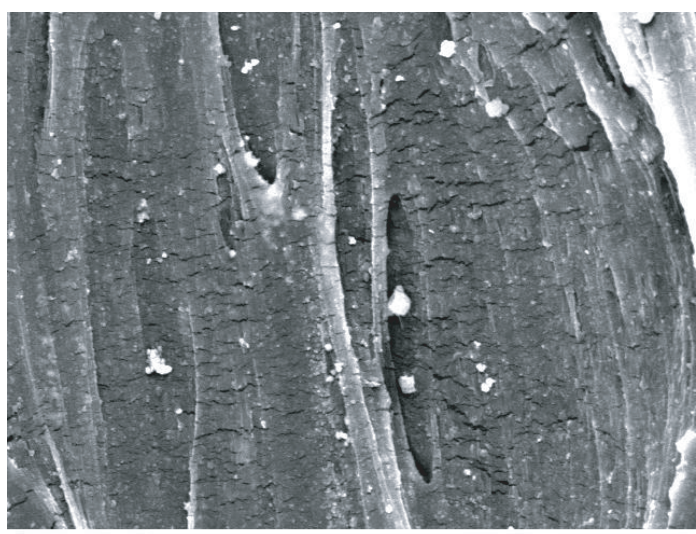

$10 \mu \mathrm{m}$

Fig. 11. Présence d'oxydes entre les filaments.

zones issues de la fissuration. Pour comprendre le mode de formation de ces zones, il faut identifier le ou les dommages qui permettent à la fissure de se propager. Il faut donc connaître l'état microstructural de la matière qui précède le front de fissure. Pour cela, nous proposons de réaliser, pendant l'observation au MEB, des microdécoupes perpendiculaires au sens d'étirement dans le fond de fissure.

\subsection{Protocole expérimental}

L'essai de fatigue, qui a permis l'apparition puis la propagation de la fissure, nous garantit la présence de dommages en fond de fissure. Il faut donc, après avoir étiré l'éprouvette, entailler le fond de fissure et découvrir ainsi les zones endommagées par la fatigue. À l'échelle mésoscopique à laquelle nous souhaitons réaliser les observations, l'utilisation d'un outil de découpe de laboratoire est exclue. En revanche, il est possible d'apporter, in situ, suffisamment d'énergie pour rompre les ponts sulfures entre les macromolécules constituant le fond de fissure et ainsi engendrer une micro-découpe. Deux sources d'énergie sont utilisées simultanément. La première, de nature mécanique, consiste à étirer l'éprouvette à 1,67 , c'est-à-dire un étirement supérieur à celui appliqué lors du chargement cyclique. La seconde consiste à concentrer le faisceau électronique sur la zone d'observation. L'apport d'énergie nécessaire par ce second moyen est difficilement quantifiable, puisque le nombre et la nature des liaisons ainsi que le nombre de macromolécules se trouvant dans la zone à découper ne sont pas connus. Ceci étant dit, l'expérience que nous avons acquise sur cette technique, nous permet de paramétrer correctement la puissance du faisceau d'électrons.

La zone propice à la fissuration est a priori la zone la plus étirée; il est donc important de pouvoir la différencier des zones moins étirées. Ceci est possible par le biais de la métallisation. En effet, puisque les déformations sont importantes, la couche d'or déposée en surface ne peut pas se déformer autant que l'élastomère. Les zones ainsi découvertes vont fortement se charger en électrons, et elles seront facilement identifiables par contraste. L'intérêt est double : à la fois mettre en évidence les parties utiles du front de fissure et permettre de concentrer de l'énergie par le faisceau d'électrons sur une zone mécaniquement favorable à la fissuration.

L'éprouvette ayant été préalablement cyclée à une élongation de 1,33 , il convient de s'assurer que les phénomènes sont les mêmes en fond de fissure lorsque nous l'étirons à 1,67. Pour ce faire, nous avons comparé zone à zone les faciès de rupture des éprouvettes testées aux deux élongations. Dans les deux cas, les évènements caractéristiques sont de même nature et se situent aux mêmes endroits sur le faciès.

\subsection{Résultats}

La figure 12 montre le fond de fissure avant et après la micro-découpe. La zone de découpe est encerclée sur la figure $12 \mathrm{~b}$. La découpe, de l'ordre du dixième de millimètre, a été pratiquée dans la partie du fond de fissure la plus étirée.

Les clichés de la figures 13 et 14 sont des agrandissements successifs de la zone fissurée propice à la découpe puisque des micro-fissures sont déjà présentes. La figure 15a montre qu'un oxyde est présent dans une zone ellipsoïdale découverte par la micro-fissure. La concentration du faisceau sur la zone fléchée de la figure 15a a permis d'initier une micro-fissure qui s'est propagée de droite à gauche en découvrant une cavité indiquée sur la figure 15b. En maintenant le faisceau concentré dans cette zone, de nouvelles fissures sont apparues et se sont propagées. Dans tous les cas, les fissures s'ouvrent sur des cavités qui contiennent pour la plupart un oxyde. Pour finir, de la figure 15b à la figure $15 \mathrm{~d}$, le faisceau électronique a été concentré de manière à provoquer la coalescence de notre découpe avec une fissure existante.

Ces observations montrent que lors de l'essai de fatigue en traction relaxante, le mécanisme d'endommagement permettant la propagation de fissure est la cavitation qui résulte de la décohésion de la matrice et des oxydes dans la zone précédant le front. Les parois des 

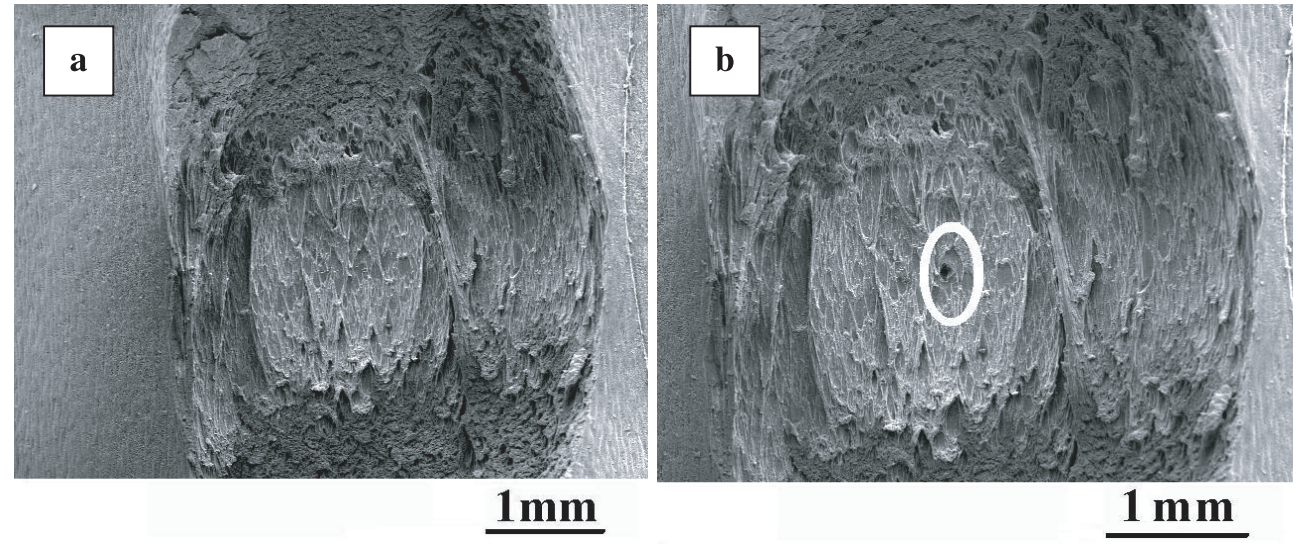

Fig. 12. (a) Fond de fissure avant découpe; (b) fond de fissure après découpe.

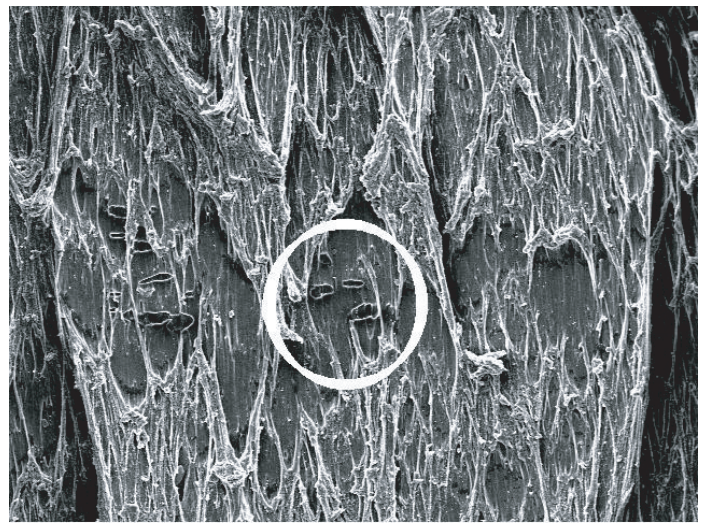

$500 \mu \mathrm{m}$

Fig. 13. Zone propice à la découpe.

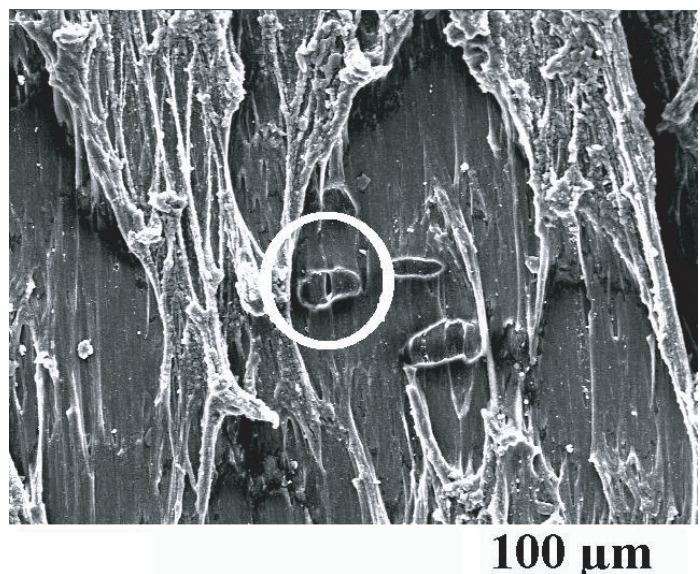

Fig. 14. Grossissement de la zone cerclée de la figure 13.

cavités découvertes sont peu à peu étirées et participent à la formation des filaments. De part et d'autre des filaments formés, il reste des parties planes correspondant au « fond $»$ des cavités découvertes. Au cours de l'essai cyclique, les filaments les plus étirés cèdent et se relaxent. La figure 16 montre une zone située au pied des ligaments. La zone A correspond aux filaments et la zone B correspond à la base des filaments. La zone B est relaxée puisque chaque arrachement de cette zone est en fait un filament qui a rompu et qui s'est rétreint.

Il faut noter que, lorsque le faisceau électronique est concentré sur les filaments fortement étirés, ces derniers ne se rompent pas, même si le temps d'exposition au faisceau est augmenté. Étant donné que la puissance du faisceau électronique demeure inchangée pendant l'observation, cela semble indiquer que la microstructure des filaments est différente de celle des zones qui les séparent.

\section{Conclusion}

Une technique de micro-découpe du fond de fissure au sein d'un MEB a été développée et utilisée afin d'observer l'endommagement de la matière devant le fond de fissure. Nous avons ainsi pu montrer que la cavitation est le dommage prépondérant dans la propagation de fissure de fatigue dans les caoutchoucs naturels chargés aux noirs de carbone et sollicités en traction relaxante. En fait, le front de fissure avance en ouvrant les parois des cavités. Fort de ces résultats, il semble désormais possible de comprendre la contribution de la cavitation au mécanisme d'avancée de la fissure. Les résultats de nos travaux en cours fourniront prochainement un mécanisme de propagation et sa modélisation. Les modèles de cavitation existants $[13,14]$ ainsi qu'une meilleure connaissance du mécanisme de fissuration permettent d'envisager la construction de modèles macroscopiques de ce type de dommage utilisables dans les codes de calculs par éléments finis. À plus long terme, l'expérience acquise devrait permettre d'améliorer la prédiction de la durée de vie en fatigue des pièces industrielles.

Remerciements. Nous remercions pour leur participation Pierre Charrier et Elisabeth Ostoja-Kuczynski de la société Trelleborg. Nous remercions également le CETIM de Nantes pour sa collaboration lors des premières observations. 

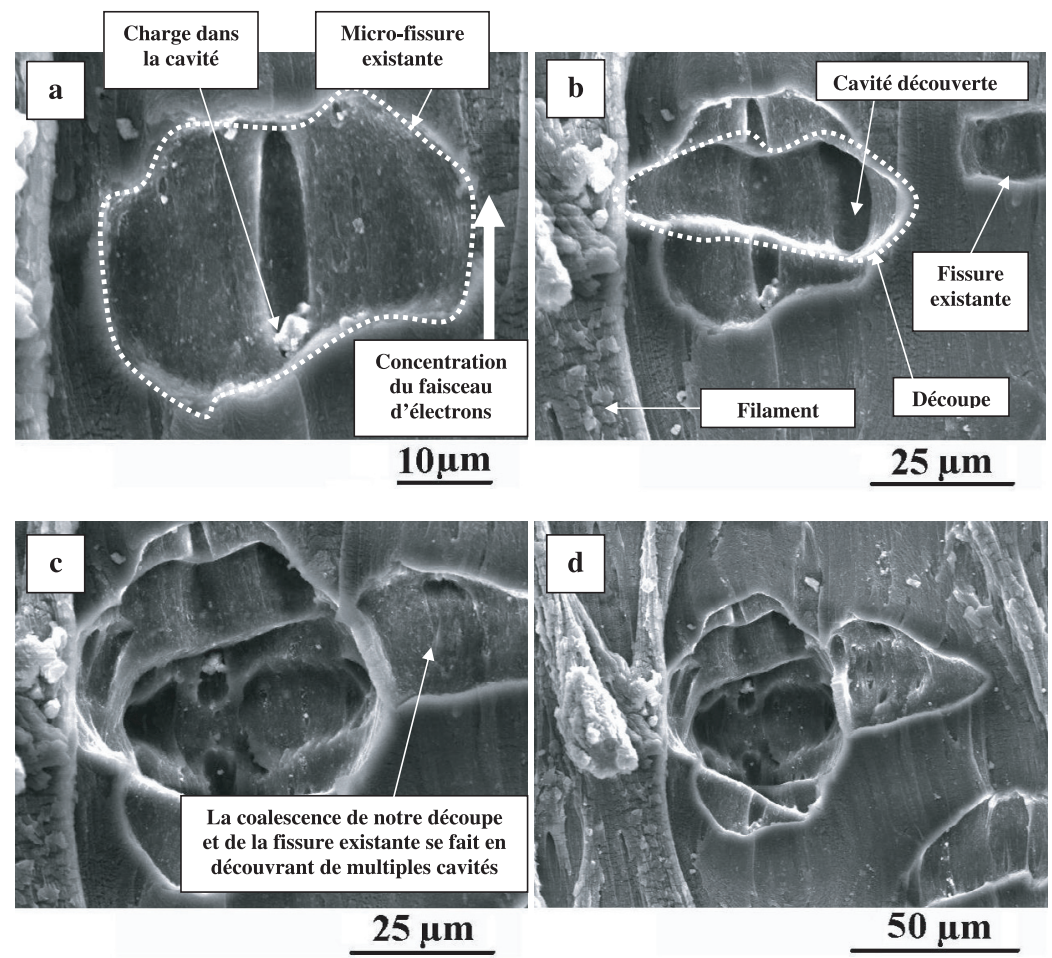

Fig. 15. (a) Départ de la découpe, formation d'une fissure; (b) coalescence de deux fissures ; (c) la fissuration continue dans l'épaisseur de l'éprouvette; (d) dernier cliché avant le cliché de la figure 12b.

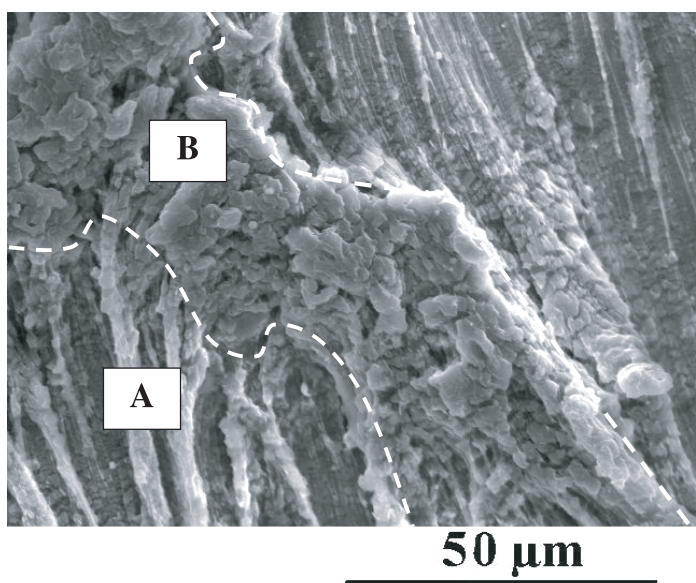

Fig. 16. Base des filaments.

\section{Références}

[1] R.S. Rivlin, A.G. Thomas, Rupture of rubber. I. Characteristic energy for tearing, J. Polym. Sci. 10 (1953) 291-318

[2] A.S. Argon, M.M. Salama, Growth of crazes in glassy polymers, Philo. Mag. 36 (1977) 1217-1234

[3] C.Y. Hui, A. Ruina, C. Creton, E.J. Kramer, Micromechanics of crack growth into a craze in a polymer glass, Macromolecules 25 (1992) 3948-3955

[4] Y. Sha, C.Y. Hui, A. Ruina, E.J. Kramer, Detailed simulation of craze fibril failure at a crap tip in a glassy polymer, Acta Mater. 45 (1997) 3555-3563
[5] M.G.A. Tijssens, E. Van Der Giessen, A possible mechanism for cross-tie fibril generation in crazing of amorphous polymers, Polymer 43 (2002) 831-838

[6] N.R. Choudhury, A.K. Bhowmick, Micromechanism of failure of thermoplastic rubber, J. Mater. Sci. 25 (1990) $2985-2989$

[7] Y. Fukahori, Fractography and fracture mechanics in rubber, in Fractography of rubber materials, A.K. Bhowmick, S.K. De (éd.), Elsevier Applied Science, London and New York, 1991

[8] A.N. Bhowmick, Quantitative fractography of rubber by image processing technique, Rubber Chem. Technol. 68 (1995) 132-135

[9] N. André, G. Cailletaud, R. Piques, Haigh Diagram for Fatigue Crack Initiation Prediction of Natural Rubber Components, Kaut. Gummi Kunstst. 52 (1999) 120-123

[10] A. Robisson, Comportement visco-hyperélastique endommageable d'élastomères SBR et $\mathrm{PU}$ : Prévision de la durée de vie en fatigue, Thèse, École Nationale Supérieure des Mines de Paris, Évry, France, 2000

[11] J.R. Beatty, Fatigue of Rubber, Rubber Chem. Technol. 37 (1964) 1341-1364

[12] J.-B. Le Cam, E. Ostoja-Kuczynski, P. Charrier, E. Verron, L. Gornet, F. Pérocheau, C. Briançon, Influence de la fréquence de sollicitation sur le faciès de rupture de pièces élastomères en fatigue, $38^{\mathrm{e}}$ Colloque annuel de Rhéologie, Physique-Chimie des produits formulés, Brest, France, du 14 au 17 octobre 2003

[13] A.N. Gent, Cavitation in rubber: a cautionary tale, Rubber Chem. Technol. 63 (1990) G49-G53

[14] C.O. Horgan, D.A. Polignone, Cavitation in nonlinearly elastic solids: a review, Appl. Mech. Rev. 48 (1995) 471-485 